\title{
Anidación e incubación artificial de huevos de tortuga golfina Lepidochelys olivacea (Testudines: Cheloniidae)
}

\section{Nesting and egg Incubation of the olive RIDLEY turtle Lepidochelys olivacea (Testudines: Cheloniidae)}

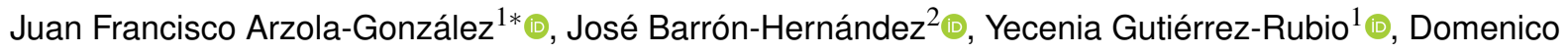 \\ Voltolina $^{3 \dagger}$, Jorge Saúl Ramírez-Pérez ${ }^{1}$ id \\ ${ }^{1}$ Facultad de Ciencias del Mar, Universidad Autónoma de Sinaloa. Ap. Postal 610, CP. 80000. Mazatlán, Sinaloa, México. \\ ${ }^{2}$ Programa Tortugas Marinas, Acuario Mazatlán. Ap. Postal 770, CP. 82017. Mazatlán, Sinaloa, México. \\ ${ }^{3}$ Centro de Investigaciones Biológicas del Noroeste, Laboratorio UAS-CIBNOR. Ap. Postal 1132, CP. 23096. Mazatlán, Sinaloa, México. \\ *Autor de correspondencia: farzola@uas.edu.mx
}

Nota científica recibida: 25 de agosto de 2018 aceptada: 24 de junio de 2019

RESUMEN. Se determinó la frecuencia de anidación y el porcentaje de eclosión en nidos artificiales de tortuga golfina (Lepidochelys olivacea) colectados en diferentes playas (Cerritos, Zona Dorada, Malecón, Pinitos, Olas Altas) de la bahía de Mazatlán, México. Se utilizaron cajas de poliuretano para la incubación artificial de nidos. Se obtuvo una eclosión promedio de crías del 75.2\% ( \pm 19.3 ). Para evitar la depredación de huevos es importante mantener la incubación artificial en cajas de poliuretano. Por la mayor incidencia de nidos en las playas Malecón ( $75.5 \pm 12.4$ nidos playa año) y Cerritos (51.3 \pm 26.5 nidos playa año), y por 11.5 y 8.3 nidos $\mathrm{km}^{-1}$, respectivamente, es necesario incrementar su vigilancia.

Palabras clave: Incubación, nidos, tortuga marina.

ABSTRACT. The frecuency of nesting and percentage of hatching eggs in artificial nests collected of Olive Ridley Sea Turtle (Lepidochelys olivacea) in several in beaches (Cerritos, Zona Dorada, Malecon, Pinitos, Olas Altas) of Mazatlan bay, Mexico were analyzed. Boxes of polystyrene were used artificial incubation of nests. Hatching success was $75.2 \%( \pm 19.3)$ and to avoid predation of eggs, incubation is required nests in boxes of polystyrene. For the higher of nests on beaches Malecon (75.5 \pm 12.4 nests beach year) and Cerritos ( $51.3 \pm 26.5$ nests beach year), and by 11.5 y 8.3 nests km ${ }^{-1}$, respectively, need to increase their vigilance.

Key words: Incubation, nests, sea turtle.

\section{INTRODUCCIÓN}

De acuerdo a la lista roja de especies amenazadas de la Unión Internacional para la Conservación de la Naturaleza (IUCN 2012), todas las especies de tortugas marinas (Caretta caretta, Eretmochelys imbricata, Chelonia mydas, Chelonia agassizii, Dermochelys coriacea, Lepidochelys kempii y Lepidochelys olivacea) se encuentran clasificadas en alguna categoría como especies en peligro (C. caretta y C. mydas), críticamente en peligro (E. imbricata y $L$. kempii) y vulnerables $(D$. coriacea y L. olivacea). En México, a partir de mayo de 1990 la captura y comercialización de tortugas marinas se encuentra prohibida, además, estas especies están protegidas en la categoría de especies en peligro de extinción por la Norma Oficial Mexicana NOM-059SEMARNAT-2010 (SEMARNAT 2010).

Como una medida de protección, durante la temporada de anidación de las tortugas marinas en las principales playas del Pacífico mexicano: Sinaloa, Nayarit, Jalisco, Colima, Michoacán y Oaxaca (García-Romero et al. 2007), los sitios de anidación son vigilados para evitar la depredación o destrucción de los nidos por diversos animales (crustáceos, coyotes, aves, entre otros), pero sobre todo por el hombre (saqueo de huevos y hembras desovando), debido a que la demanda ilegal de 
carne, aceites y huevos de tortugas marinas se ha incrementado en los últimos años (Bárcenas y Maldonado 2009). En la costa de Sinaloa existen cinco campamentos para la protección y conservación de las tortugas marinas a cargo de diversas instituciones del gobierno federal (El Verde Camacho -Comisión Nacional Áreas Naturales Protegidas CONANP-), gobierno municipal (Acuario Mazatlán), universidad (Ceuta y Caimanero -Universidad Autónoma de Sinaloa) y empresas privadas (Isla de la Piedra), realizan esfuerzos para conservar y proteger este recurso. En algunas bahías de Sinaloa se ha estudiado la influencia de la humedad, temperatura y la granulometría en la incubación de nidos in situ comparados con nidos artificiales en cajas de poliuretano en la playa El Verde Camacho, al norte de Mazatlán, con diferencias en la eclosión principalmente por la humedad entre nidos naturales y artificiales (ArzolaGonzález 2007). En otras playas, se ha estudiado la incubación de huevos de tortuga $L$. kempii en cajas convencionales (poliuretano) y cajas de madera con hule espuma, resultando mejor el porcentaje de eclosión en estas últimas cajas en $95.6 \%$, que las cajas de poliuretano (Vázquez-Sauceda et al. 2008). Debido a la poca información sobre los sitios de anidación de la tortuga golfina en las diversas playas de la bahía Mazatlán, el objetivo del presente estudio fue determinar los sitios de anidación más importantes a lo largo de la bahía, además del porcentaje de eclosión y de huevos interrumpidos (fase embrionaria) en nidos incubados de forma artificial en cajas de poliuretano.

\section{MATERIALES Y MÉTODOS}

Las playas de la bahía de Mazatlán comprenden una longitud de aproximadamente $21 \mathrm{~km}\left(23^{\circ}\right.$ $11^{\prime} 20^{\prime \prime}$ y $23^{\circ} 15^{\prime} 17^{\prime \prime} \mathrm{LN}$ y $106^{\circ} 24^{\prime} 59^{\prime \prime}$ y $106^{\circ} 29^{\prime}$ $41^{\prime \prime}$ LO). Las playas, en ocasiones se encuentran separadas por diversas áreas rocosas o por espacios artificiales (construcciones) como consecuencia del desarrollo urbano del Puerto de Mazatlán. La playa donde comúnmente desovan las hembras de tortuga golfina se dividió en cinco secciones de longitud variable, siendo de norte a sur: Cerritos (6.15 $\mathrm{km})$, Zona Dorada (5 km), Malecón (6.55 km), Pinitos $(0.15 \mathrm{~km})$ y Olas Altas $(2.55 \mathrm{~km})$.

Los nidos de L. olivacea se localizaron durante las temporadas de anidación (junio a octubre). Las playas se vigilaron de 20:00 a 06:00 horas con una motocicleta todo terreno. Los huevos de cada nidada se recolectaron y depositaron en bolsas de plástico y se trasladaron a la cámara de incubación del Acuario Mazatlán donde se sembraron en cajas de poliuretano de 40 de largo x 30 de ancho y $30 \mathrm{~cm}$ de altura, la base y la tapa se perforaron para el intercambio gaseoso entre los embriones y el medio. Las cajas contenían una primera capa de arena de aproximadamente $10 \mathrm{~cm}$, después se colocaron todos los huevos procurando no sobrepasar más de tres hileras, para luego recubrir las nidadas con una segunda capa de arena de entre 10 y $15 \mathrm{~cm}$. Cada caja se cerró y se etiquetó con los datos de campo (fecha, hora, lugar y cantidad de huevos). Posteriormente, las cajas con las nidadas se colocaron en estantes de madera dentro de la cámara de incubación a temperatura de 32 ${ }^{\circ} \mathrm{C} \pm 1$.

El tiempo entre la colecta de las nidadas y su incubación artificial fue de 1 y $2 \mathrm{~h}$, dependiendo de la distancia de la playa. Para la determinación de la humedad de la arena en las cajas de incubación se utilizaron $100 \mathrm{~g}$ de arena de cada sitio que se utilizó para la incubación de los nidos, la arena se colocó en charolas de aluminio previamente pesadas en una balanza digital $( \pm 0.1 \mathrm{~g})$, y se depositaron en un horno a $100{ }^{\circ} \mathrm{C}$ por $24 \mathrm{~h}$, después se volvieron a pesar las charolas con la arena seca para obtener con las diferencias en peso, la cantidad de humedad pérdida, Para mantener la humedad cada 15 días se suministró agua por aspersión para mantener la humedad perdida durante la incubación.

Después del período de incubación (días), se obtuvo el porcentaje de eclosión y de embriones no desarrollados (fase I, II, III, IV y HI), y la frecuencia de ubicación del nido (n). La determinación de fases embrionarias se determinó de acuerdo con los criterios de Arzola-González (2007), las fases I, II y III, corresponde a embriones en desarrollo con diferentes tallas, mientras que la fase IV corresponde a las crías que no alcanzaron a emerger de la nidada, 
y $\mathrm{HI}$ a huevos no fecundados.

Para estimar las diferencias estadísticas entre el número de nidos, nidos por kilómetro entre las playas, huevos (número), crías (número), incubación (días) y eclosión (\%), se realizaron pruebas de normalidad (Lilliefords), homoscedasticidad (Bartlett) y se aplicaron pruebas de Kruskal-Wallis. Al resultar significativos, se realizaron pruebas de comparaciones múltiples (Dunn). En todos los casos se utilizó un nivel de significancia de $p<0.05$ (Zar 2009).

\section{RESULTADOS Y DISCUSIÓN}

Se incubaron los huevos recolectados en 637 nidos con promedio de 88.7 ( \pm 21.6 ) huevos por nido (55 798 huevos), y se obtuvieron 41753 crías lo que indica un porcentaje de eclosión del $75.2 \%$. En promedio el tiempo de incubación fue 46.1 ( \pm 5.8 ) días, con variación mínima de 45 y máxima de 48 días. La mortalidad total (24.8\%) fue de 8195 crías muertas (57.8\%) y 5850 (41.4\%) embriones que no completaron su desarrollo (Tabla 1). El porcentaje de eclosión observado fue superior al $47 \%$ señalado por Arzola-González (2007) para L. olivacea y similar al $78 \%$ reportado por García-Romero et al. (2007) en nidos de tortugas marinas (L. olivacea, C. caretta y D. coriacea). Al respecto, estudios con cajas de poliuretano para la incubación de huevos de tortugas marinas, reportan para $L$. kempii un porcentaje superior al 79.9\% (Vázquez-Sauceda et al. 2008), mientras que para $L$. olivacea se reporta un $89.6 \%$, de eclosión (López-Correa et al. 2010).

Es importante resaltar que los esfuerzos de conservación de nidos y de hembras en desove indican que las nidadas de L. olivacea se mantiene estable en algunas playas (Malecón), en otras disminuyen (Cerritos y Zona Dorada), pero también incrementa el número de nidos en el tiempo. En nidos recolectados de tortuga golfina, la zona del Malecón tuvo el mayor número de nidos $(n=302)$ y los menores en las playas Olas Altas $(n=40)$ y Pinitos $(n=0)$ (Tabla 1). Es muy probable que en Pinitos la ausencia de nidos se deba a que durante la bajamar quedan expuestos numerosos promontorios rocosos, de tal forma que el perfil de playa se modifica constantemente, lo que ocasiona que las hembras no encuentren un área para el desove. Esto coincidió con lo observado por Rincón-Díaz y RodríguezZarate (2004), quienes indicaron que en playas rocosas disminuye la probabilidad de desove. Pero debido a su ubicación entre las playas del Malecón y Olas Altas, es necesario continuar con la vigilancia de la playa dentro de la franja costera de la bahía.

Es evidente que algunos sitios de playa de la bahía Mazatlán representan una oportunidad de desove para las hembras de tortuga golfina, siendo las playas Malecón y Cerritos las que tuvieron el mayor índice de nidos, con 11.5 y 8.3 nidos $\mathrm{km}^{-1}$, respectivamente; por lo que se recomienda una mayor incidencia de recorridos en dichas playas para la conservación de los nidos de esta especie. Aunque, por su densidad de nidos por kilómetro, los restantes sitios de desove y anidación (Zona Dorada $=4.5$ y Olas Altas $=3.9$ nidos $\mathrm{km}^{-1}$ ) deben continuar monitoreándose las próximas temporadas de reproducción para establecer si la densidad de nidadas en cada sitio, se mantiene, incrementa o disminuye.

Para los embriones interrumpidos, la fase III fue la más crítica con $41.5 \%$, las mínimas fueron en $\mathrm{HI}$ (huevos inflados) y fase I con 9.6 y $9.3 \%$, respectivamente; aunque entre años sucesivos disminuyeron los mayores porcentajes de embriones interrumpidos (Tabla 2). El mayor porcentaje de embriones no desarrollados coincidió con ArzolaGonzález (2007) en nidos de L. olivacea en cajas de poliuretano. Sin embargo, el éxito de eclosión promedio obtenido se pondera cuando algunos factores involucrados en la interrupción de embriones sean, entre otros, la distribución de los huevos ubicados en las capas inferiores del nido, los cuales posiblemente presentan una menor disponibilidad del oxígeno y por consiguiente, incrementó el riesgo de los embriones a perecer por asfixia, aunado a lo anterior, estos embriones deben soportar la presión de los huevos de las capas superiores. Además, la mortalidad de los embriones en fase IV, probablemente fue provocada por un exceso de humedad (Foley et al. 2006). Por lo que estos aportes probablemente ocasionen una compactación de cáscaras y huevos interrumpidos dentro de las cajas impidiendo la salida 
Tabla 1. Número de nidos y número de nidos $\mathrm{km}^{-1}$ de $L$. olivacea por línea de playa, promedio y desviación estándar $( \pm \mathrm{DE})$ en playas de la bahía de Mazatlán. $\mathrm{CE}=$ Cerritos, ZD = Zona Dorada, MA $=$ Malecón, $\mathrm{PI}=$ Pinitos, $\mathrm{OA}=$ Olas Altas.

\begin{tabular}{ccccccccccc}
\hline \multicolumn{10}{c}{ Nidos Playa Años } \\
\hline \multicolumn{10}{c}{ Nidos km kn $^{-1}$} \\
\hline Año & CE & ZD & MA & PI & OA & CE & ZD & MA & PI & OA \\
2001 & 85 & 30 & 81 & 0 & 9 & 13.8 & 6.0 & 12.4 & 0 & 3.5 \\
2002 & 59 & 15 & 57 & 0 & 3 & 9.6 & 3.0 & 8.7 & 0 & 1.2 \\
2003 & 32 & 23 & 84 & 0 & 4 & 5.7 & 4.6 & 12.8 & 0 & 1.6 \\
2004 & 65 & 22 & 80 & 0 & 24 & 4.2 & 4.4 & 12.2 & 0 & 9.4 \\
Media & $51.3^{b c}$ & $22.5^{a b}$ & $75.5^{c}$ & 0 & $10^{a}$ & $8.3^{a b}$ & $4.5^{a}$ & $11.5^{b}$ & 0 & $3.9^{a}$ \\
DE & \pm 26.5 & \pm 6.11 & \pm 12.4 & \pm 0 & \pm 9.7 & \pm 4.3 & \pm 1.2 & \pm 1.9 & \pm 0 & \pm 3.8
\end{tabular}

Diferentes letras indican diferencias significancias entre los valores medios (prueba de Kruskal-Wallis, $\alpha=0.05 ; a \leq a b \leq b$ y $a<b)$.

Tabla 2. Total, máxima, media, desviación estándar (n) y porcentaje (\%) de huevos, crías, incubación (días), supervivencia, fases embrionarias I, II, III, IV y V, en nidos de tortuga marina L. olivacea.

\begin{tabular}{|c|c|c|c|c|c|c|c|c|c|}
\hline \multirow[t]{2}{*}{ Año } & \multirow[t]{2}{*}{ Huevos $(\mathrm{n})$} & \multirow[t]{2}{*}{ Crías } & \multirow[t]{2}{*}{ Incubación (días) } & \multirow[t]{2}{*}{ Eclosión (\%) } & \multicolumn{5}{|c|}{ Fases embrionarias ( $n$ ) } \\
\hline & & & & & I & II & III & IV & $\mathrm{HI}$ \\
\hline 2001 & $91.3^{a}$ & $66.7^{a b}$ & $47.4^{b}$ & $71.6^{c}$ & 270 & 515 & 1,090 & 395 & 130 \\
\hline 2002 & $88.6^{a}$ & $60.8^{a}$ & $44.7^{a}$ & $66^{a}$ & 219 & 249 & 1,083 & 478 & 213 \\
\hline 2003 & $86.8^{a}$ & $75^{b}$ & $47.3^{b}$ & $84.2^{b}$ & 6 & 37 & 189 & 330 & 55 \\
\hline 2004 & $88.0^{a}$ & $71^{b}$ & $44.9^{a}$ & $79.3^{c}$ & 0 & 1 & 66 & 313 & 163 \\
\hline Total & 55,798 & 41,753 & * & * & 543 & 802 & 2,428 & 1,516 & 561 \\
\hline Máxima & 155 & 135 & 64 & 100 & * & * & * & * & * \\
\hline Media & 88.7 & 68.4 & 46.1 & 75.2 & 135.8 & 200.5 & 607 & 379 & 1403 \\
\hline D.E. & 21.6 & 23.7 & 5.8 & 19.9 & & & & & \\
\hline Porcentaje (\%) & & & & & 9.3 & 13.7 & 41.5 & 25.9 & 9.6 \\
\hline
\end{tabular}

de crías con facilidad de la nidada (Brenes 2011). Los huevos sin aparente desarrollo (HI) fue de $9.6 \%$, quizás este mínimo porcentaje fue provocado por la infertilidad que ha sido determinada previamente por la ausencia de un embrión visible en huevos de tortugas marinas (Caut et al. 2006), C. mydas (Fowler et al. 1979), L. olivacea (Arzola-González 2007) y D. coriacea (Velázquez et al. 2014).

Por otro lado, la humedad y temperatura durante la incubación repercuten en la viabilidad de los embriones, ya que su único medio de protección es la arena (Sandoval et al. 2010). Algunos autores indican que la arena para la incubación contenga aproximadamente $0.35 \mathrm{~kg}$ de agua por cada $5 \mathrm{~kg}$ de arena (López-Correa et al. 2010), en el presente estudio durante la incubación en las cajas de poliuretano solo se proporcionó agua por aspersión con la finalidad de recuperar la humedad perdida principalmente por la evaporación, pero la incubación de huevos es afectada por condiciones de humedad en exceso o mínimas, constituyendo una de las principales causas de mortalidad de embriones y crías de tortuga marina
(Foley et al. 2006, Brenes 2011).

El porcentaje de embriones interrumpidos, puede ser considerado positivo si se justifica que las nidadas se encuentran en peligro de ser depredadas. Por lo que las playas Malecón y Cerritos por su alta incidencia de nidos y hembras adultas, requiere de mayor vigilancia. Es eficiente la utilización de las cajas de poliuretano como alternativa de incubación de huevos de tortuga golfina, además considerando que L. olivacea es una especie en peligro de extinción, el porcentaje de eclosión del $75.2 \%$ obtenido, es de interés para la protección y conservación de los sitios de anidación de la tortuga golfina en las diferentes playas de la bahía Mazatlán.

\section{AGRADECIMIENTOS}

Al personal del Cuerpo Académico Consolidado Manejo de Recursos Pesqueros (UASCA-2104), al Acuario Mazatlán por los recursos otorgados y a SEMARNAT (SGPA/DGVS/009235/18) por permitir la extracción de nidos para su incubación ar- 
tificial de tortugas marinas de la bahía Mazatlán.

\section{LITERATURA CITADA}

Arzola-González JF (2007) Humedad y temperatura en nidos naturales y artificiales de tortuga golfina Lepidochelys olivacea. Revista de Biología Marina y Oceanografía 42: 377-383.

Bárcenas IA, Maldonado GA (2009) Malformaciones en embriones y neonatos de tortuga golfina Lepidochelys olivacea en Nuevo Vallarta, Nayarit, México. Veterinaria México 40: 371-380.

Brenes O (2011) Efecto de la precipitación en el proceso de la incubación de las nidadas de tortuga lora. Revista de Ciencias Ambientales 41: 27-36.

Caut S, Guirlet E, Jouquet P, Girondot M (2006) Influence of nest location and yolkless eggs on the hatching success of leatherback turtles clutches in French Guiana. Canadian Journal of Zoology 84: 908-915.

Foley AM, Peck SA, Harman GR (2006) Effects of sand characteristic and inundation on the hatching success of loggerhead sea turtle (Caretta caretta) clutches on low-relief mangrove islands in southwest Florida. Chelonian Conservation and Biology 5: 32-41.

Fowler L (1979) Hatching success and nest predation in the green sea turtle, Chelonia mydas at tortuguero, Costa Rica. Ecology 60: 946-955.

García-Romero M, Hernández-Dueñas DL, García-Castañeda B, Santos-Soto A, Meyer-Willerer A (2007) Protección y conservación de tortugas marinas de la zona costera de Michoacán, México. Avances en Investigación Agropecuaria 11: 15-22.

IUCN (2012) IUCN Red List Categories and Criteria: Version 3.1. Second Edition. Species Survival Commission. IUCN, Gland, Switzerland and Cambridge, UK 32P. http://www.iucnredlist.org/technical-documents/categories-and-criteria/2001-categories-criteria. Fecha de consulta: 9 de abril de 2018.

López-Correa J, Porta-Gámdara M, Gutiérrez J, Gómez-Muñoz V (2010) A novel incubator to simulate natural thermal environment of sea turtle eggs. Journal of Thermal Biology 35: 138-142.

Rincón-Díaz MP, Rodríguez-Zarate CJ (2004) Caracterización de playas de anidación de tortugas marinas en el archipiélago de San Bernardo, Caribe Colombiano. Boletín de Investigaciones Marinas y Costeras 33: 137-158.

Sandoval S, Gómez,-Muñoz V, Gutiérrez J, Porta-Gámdara GM (2011) Metabolic heat estimation of the sea turtle Lepidochelys olivacea embryos. Journal of Thermal Biology 36: 138-141.

SEMARNAT (2010) NOM-059-SEMARNAT-2010. Protección ambiental-Especies nativas de México de flora y fauna silvestres-Categorías de riesgo y especificaciones para su inclusión, exclusión o cambio-. Lista de especies en riesgo. México, D.F. http://www.profepa.gob.mx/innovaportal/file/435/1/NOM_059_SEMARNAT_ 2010.pdf. Fecha de consulta: 12 de junio de 2018.

Vázquez-Sauceda M, Aguirre-Guzmán G, Pérez-Castañeda R, Sánchez-Martínez J, Martin-Del Campo R, LoredoOsti J, Rábago-Castro J (2008) Evaluación de la influencia de dos cajas de transporte de huevos sobre la incubación, eclosión y nacimiento de crías de tortuga lora Lepidochelys kempii. Ciencias Marinas 34: 101105.

Velázquez F, Prieto A, Prieto E (2014) Mortalidad embrionaria en cuatro nidos de tortuga cardón Dermochelys coriacea en playa San Luis, sector Los Chivos, Cumaná Estado Sucre. Revista Multidisciplinaria del Consejo de Investigación de la Universidad del Oriente 26: 243-248.

Zar JH (2009) Biostatistical analysis. 5th Edition. Prentice-Hall. New Jersey, USA. 960p. 
